\title{
SIMULTANEOUS MULTI-FREQUENCY IMAGING \\ OBSERVATIONS OF SOLAR MICROWAVE BURSTS
}

\author{
M. R. KUNDU, S. M. WHITE, and E. J. SCHMAHL \\ Astronomy Program, Univ. of Maryland, College Park, MD 20742, U.S.A.
}

\begin{abstract}
We review the results of simultaneous two-frequency imaging observations of solar microwave bursts with the Very Large Array. Simultaneous 2 and $6 \mathrm{~cm}$ observations have been made of bursts which are optically thin at both frequencies, or optically thick at the lower frequency. In the latter case the source structure may differ at the two frequencies, but the two sources usually seem to be related. However, this is not always true of simultaneous 6 and $20 \mathrm{~cm}$ observations. The results have implications for the analysis of non-imaging radio data of solar and stellar flares.
\end{abstract}

\section{Introduction}

The analysis of the energetics of both stellar and most solar flares relies on the interpretation of non-imaging radio and/or X-ray observations. The type of analysis is often of the following type (e.g., Batchelor et al., 1985): assuming that the radio emission at a frequency below the peak of the spectrum is optically thick thermal emission (flux $\sim A f^{2} \mathrm{~T}$ ), one uses the flux at a given frequency $f$ together with a temperature $T$ from X-ray data to calculate an area $A$. Departure from the $f^{2}$ law gives the variation of the optically-thick area with frequency, and time dependence is used to deduce the rate at which the source grows in the impulsive phase. There is an implied assumption that the structure of the source is simple, and that, in some sense, the sources one is seeing at the different frequencies are the same.

It is important to check this non-imaging technique against imaging observations. Using two sub-arrays of about 13 antennae each, the VLA* can be used to image solar microwave bursts simultaneously at two frequencies. Here we review the results of such observations from the last solar maximum. There are several different types of burst found. The simplest case is that in which the time profiles of the two frequencies are identical and the maps show a simple source at the same location at both frequencies. This situation implies that one is seeing the same electrons at both frequencies, and one can then accurately calculate the magnetic field, particle density and distribution in the source. Naturally, this is rare, but there is one good example (October 1, 1980).

A second type of burst shows similar time profiles but different locations for the two frequencies: when the high frequency emission comes from two small sources and the lower frequency from a large featureless source, this can be interpreted as footpoint plus loop-top emission, from one population of non-thermal electrons in the loop. There are other cases in which the different time profiles and different spatial locations of the

* The Very Large Array is a facility of the National Radio Astronomy Observatory, which is operated by Associated Universities, Inc., under contract with the National Science Foundation. 
sources at the two frequencies imply that one is seeing two populations of electrons, usually one thermal component and another non-thermal. Particularly at $20 \mathrm{~cm}$ $(1.4 \mathrm{GHz})$ one rarely seems to see the same population of electrons which radiates at higher frequencies. In some events the spatial structure of the source region is so complex that no simple conclusions can be drawn. In the space available here we discuss the observations briefly, and summarize the implications for analysis of non-imaging data.

\section{Simple Optically-Thin Non-Thermal Burst}

Figure 1(a) shows the time profile of the flux from a burst on October 1, 1980 (Kundu, Velusamy, and White, 1987), as observed in the VLA's shortest spacings. The time profiles at the two wavelengths, $6 \mathrm{~cm}(5 \mathrm{GHz})$ and $2 \mathrm{~cm}(15 \mathrm{GHz})$, are essentially indentical. In Figure 1(b), we plot the time evolution of the peak brightness temperature in the map during the impulsive phase of the flare, together with the degree of polarization at both wavelengths. This confirms the close resemblance of the two time profiles.

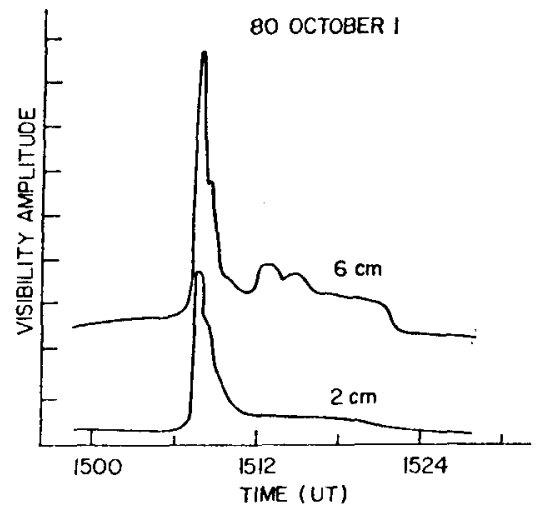

a

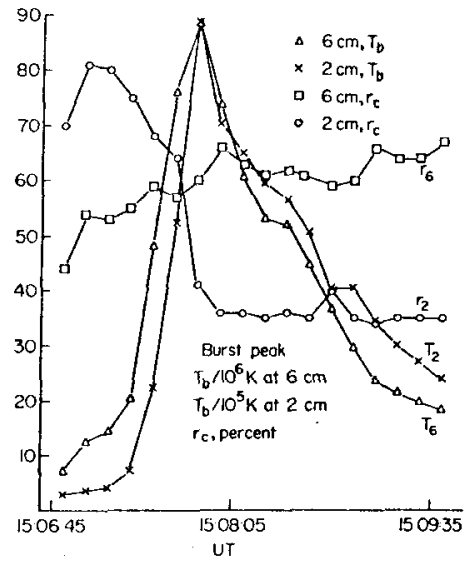

b

Fig. 1. (a) The time profile of the flux in the shortest spacings at the VLA at 2 and $6 \mathrm{~cm}$ wavelengths. (b) The time profiles of the maximum brightness temperatures in the 2 and $6 \mathrm{~cm}$ maps during the peak of the burst in (a), together with the variation of the degree of circular polarization at both wavelengths.

Figure 2 shows the structure of the pre-event region at $6 \mathrm{~cm}$ (Figure 2(a)), the 6 and $2 \mathrm{~cm}$ burst sources in $I$ (Figure $2(\mathrm{~b}, \mathrm{c})$ ), and the location of the burst source superposed on a Kitt Peak magnetogram of the region (Figure 2(d)). This indicates that the burst occurred over the neutral line between a predominantly positive magnetic field region and an intruding negative region, and strongly suggests that reconnection between the regions of opposite polarity played a role in the onset of the burst.

The spatial locations of the 6 and $2 \mathrm{~cm}$ burst sources in Figure 2, together with the similar time evolutions, suggest that we are seeing the same electrons in the same location at both wavelengths. The brightness temperature of the $6 \mathrm{~cm}$ emission indicates 

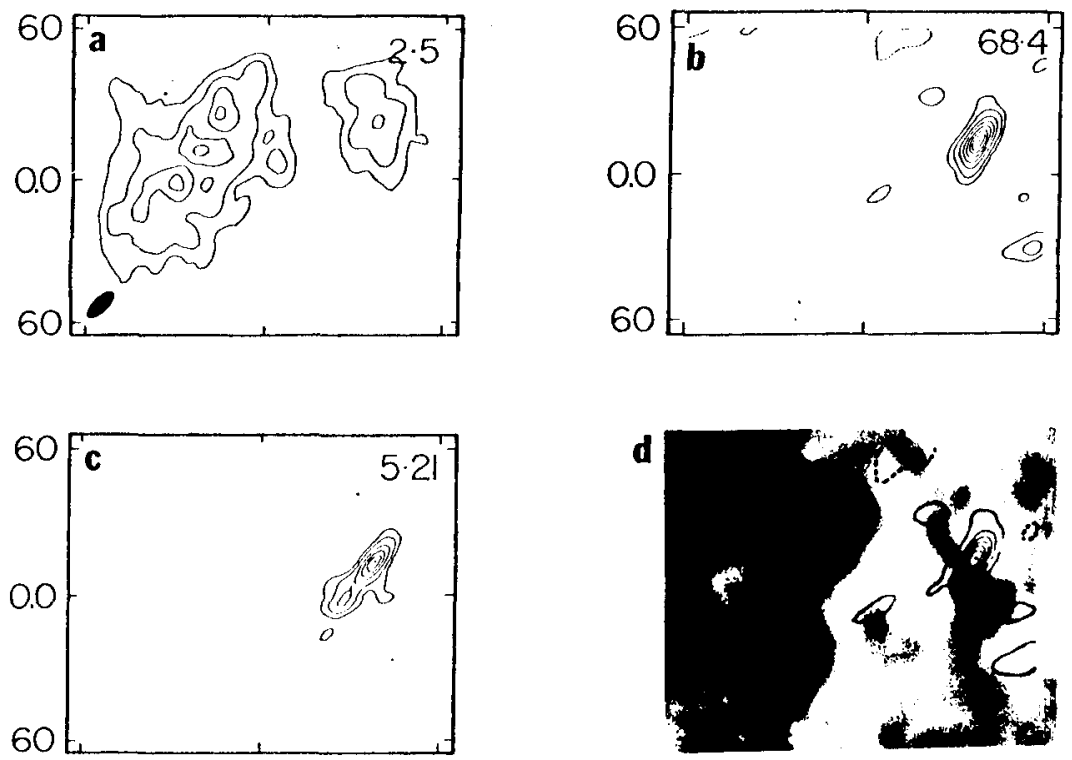

Fig. 2. (a) The pre-flare map of the active region at $6 \mathrm{~cm}$. (b, c) The $I$ maps of the burst peak at 6 and $2 \mathrm{~cm}$, respectively. (d) The Kitt Peak magnetogram of the active region, together with the contours of the burst source from (b) superposed.

non-thermal gyrosynchrotron as the likely emission mechanism. The high degree of polarization at both wavelengths, together with the spectrum of the burst obtained from patrol telescopes which gradually decreases from 2.8 to $20 \mathrm{GHz}$, suggests that both 2 and $6 \mathrm{~cm}$ are in the optically thin range. With this information the full parameters in the source region can be derived, assuming a homogeneous source: we find a surprisingly flat spectral index of 1.44 for the energy distribution of the electrons, an electron number density of $9 \times 10^{4} \mathrm{~cm}^{-3}$ (for the non-thermal radiating electrons above $10 \mathrm{keV}$ ), a total energy of about $8 \times 10^{23}$ ergs in the electrons, and a magnetic field of $390 \mathrm{G}$. We assume that the electrons are trapped in a compact low-lying loop, and that this explains the relatively high magnetic field for a $6 \mathrm{~cm}$ non-thermal source. In order for the assumption of a homogeneous source to be valid, it may be necessary to assume either that the electrons are trapped at the top of the loop due to a highly anisotropic pitch angle distribution, or that the magnetic field strength does not change greatly along the loop (which can be achieved if the loop cross-section is roughly constant). In practice, we believe that it is most likely that there is a range of magnetic fields in the loop, and that the value $390 \mathrm{G}$ is most representative of this range.

\section{Weak Burst Optically Thick at $6 \mathrm{~cm}$ and Optically Thin at $2 \mathrm{~cm}$}

In the first burst discussed the $6 \mathrm{~cm}$ emission was optically thin, since it was highly polarized and the burst spectrum from patrol telescopes indicated a spectrum decreasing from about $15 \mathrm{~cm}$ (to shorter wavelengths). Usually, however, solar microwave bursts 
have a spectral peak at around $4 \mathrm{~cm}(8 \mathrm{GHz})$, and $6 \mathrm{~cm}$ emission is then optically thick while $2 \mathrm{~cm}$ emission is optically thin. Dulk, Bastian, and Kane (1986) observed such a burst at 6 and $2 \mathrm{~cm}$ and in hard X-rays on November 22, 1981. Figure 3(a) shows the time evolution of the flux from the burst in each wavelength range, and Figure 3(b) shows the relative locations of the $2 \mathrm{~cm}$ (solid lines) and $6 \mathrm{~cm}$ (dashed lines) sources.

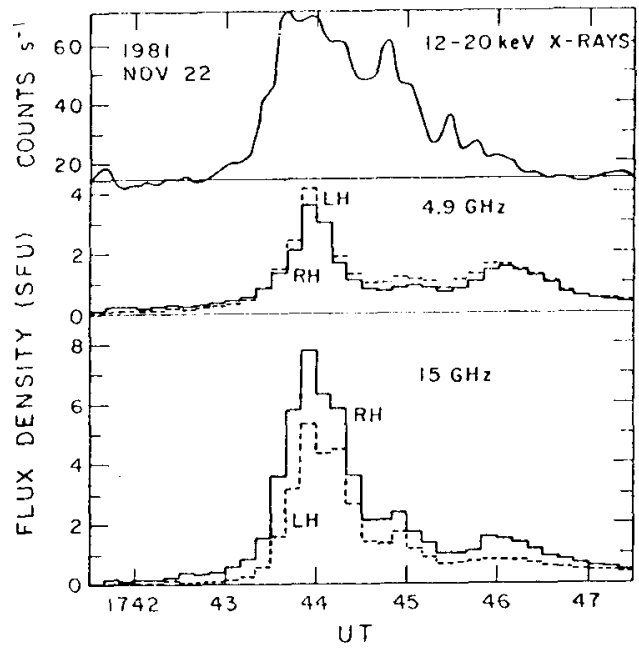

a

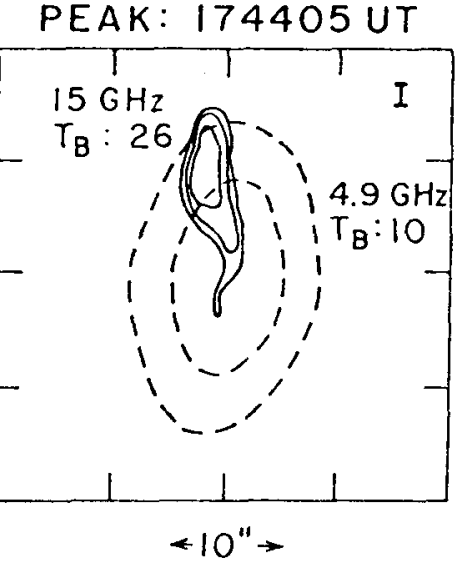

b

Fig. 3. (a) The time profiles of the hard X-ray, 6 and $2 \mathrm{~cm}$ fluxes during the burst on 1981 November 22 .

(b) The locations of the 6 and $2 \mathrm{~cm}$ burst sources.

The brightness temperature peaked at only $10^{7} \mathrm{~K}$ at $6 \mathrm{~cm}$ and $3 \times 10^{6} \mathrm{~K}$ at $2 \mathrm{~cm}$, whereas the X-ray temperature was about $2 \times 10^{7} \mathrm{~K}$. Since the optically thick $6 \mathrm{~cm}$ emission is lower in temperature than the X-rays, a simple homogeneous model does not fit these observations. This event can be modelled by a constant-density inhomogeneous source in which the temperature is greatest at the middle of a gaussianshaped region and decreases towards the edges. The emission mechanism for all three components is thermal bremsstrahlung (gyroresonance is ruled out by the spectrum, and the low brightness temperature argues against non-thermal emission). The $6 \mathrm{~cm}$ emission is optically thick throughout, while the $2 \mathrm{~cm}$ emission is optically thin. The magnetic field can be estimated from the polarization of the $2 \mathrm{~cm}$ source to be around $200 \mathrm{G}$.

We note that these authors also observed a burst at $2 \mathrm{~cm}$ which was absent at $6 \mathrm{~cm}$. They noticed that the radiation had to pass over a strong sunspot on the way to the earth, and concluded that the longer wavelength radiation was absorbed. Without the availability of imaging data showing the location of the sunspot, the interpretation of this event based on the spectrum alone would probably have been erroneous. This illustrates the potential dangers of non-imaging data. 


\section{Footpoint + Loop-Top Events}

Figure 4(a) shows the time evolution of a burst observed at 6 and $2 \mathrm{~cm}$ on 1981 November 13 (Shevgaonkar and Kundu, 1985). The $2 \mathrm{~cm}$ and hard X-ray profiles are very similar in the rise phase, and the $6 \mathrm{~cm}$ profile shows a similar shape but is delayed with respect to the other two profiles by about $20 \mathrm{~s}$. The maps (Figure 4(b) and 4(c)) show two sources interpreted as loop footpoints at $2 \mathrm{~cm}$, and a single large featureless source at $6 \mathrm{~cm}$ which is interpreted as the loop top (here by 'loop top' we mean essentially the whole of the volume in which, in the conventional loop picture, the loop turns over, and not just a small volume at the very centre of the loop). The explanation here is that in the higher magnetic fields of the footpoints the emissivity of higher frequency radiation is greater. One deduces magnetic fields in the $6 \mathrm{~cm}$ source below $200 \mathrm{G}$, and can show that the $6 \mathrm{~cm}$-emitting electrons would not be seen at $2 \mathrm{~cm}$,
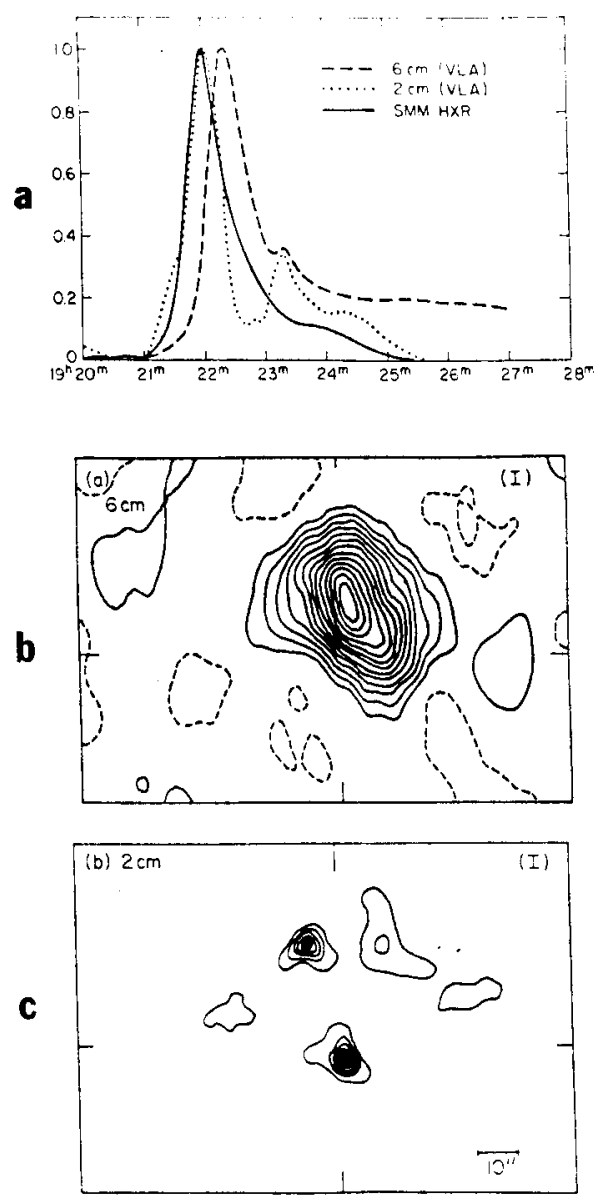

Fig. 4. (a) The time profiles of the hard X-ray, 6 and $2 \mathrm{~cm}$ fluxes of the burst on 1981 November 13, all normalized to the same peak value for comparison. (b) The $6 \mathrm{~cm}$ map of the burst source. (c) The $2 \mathrm{~cm}$ map of the burst source. 
provided that the electron energy spectral index exceeds 4 . The delay of the $6 \mathrm{~cm}$ peak relative to the $2 \mathrm{~cm}$ and hard $\mathrm{X}$-ray peaks can be due to a propagation time, or to the electrons being different populations with different production times: thus, depending on the conditions, the $6 \mathrm{~cm}$ emission could be due to thermal electrons which require longer to be produced, while the $2 \mathrm{~cm}$ electrons are non-thermal electrons produced in the impulsive phase.

\section{Simultaneous 6 and $20 \mathrm{~cm}$ Observations}

The usual spectrum of a homogeneous non-thermal gyrosynchrotron source with a field of several hundred gauss peaks at around $4 \mathrm{~cm}$, and thus the flux at $20 \mathrm{~cm}$ from such a source tends to be small. For this reason the sources seen at $20 \mathrm{~cm}$ in conjunction with a burst might be expected to show the influence of something other than the non-thermal electrons, as opposed to simultaneous 2 and $6 \mathrm{~cm}$ observations, where both wavelengths are close to the peak of the non-thermal burst spectrum. A burst on 1980 May 15 was observed at 6 and $20 \mathrm{~cm}$ by Velusamy et al. (1987) and showed complexity in both the time profile and in the mapped structure. Figure 5 shows the time profile of the event in two hard X-ray channels from ISEE-3, the GOES soft X-ray flux and

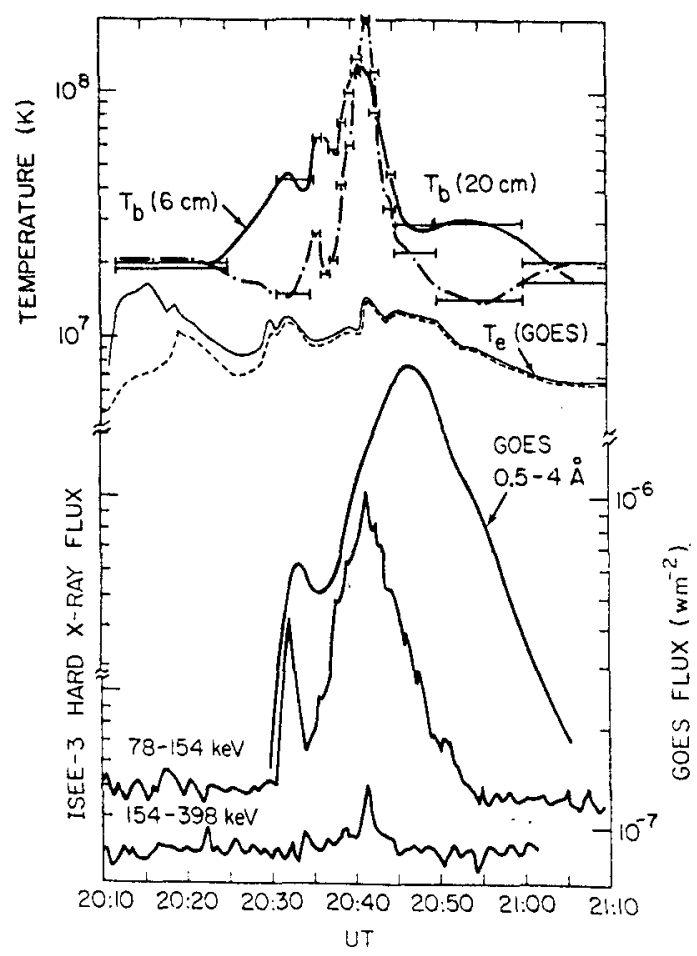

Fig. 5. The time profiles of the 2 and $6 \mathrm{~cm}$ peak brightness temperatures, the X-ray electron temperature deduced from the fluxes in two GOES soft-X-ray bands, the flux in one of the GOES bands, and the hard $X$-ray fluxes in two of the channels of the ISEE-3 spectrometer. 
electron temperature profiles, and the variation of the maximum brightness temperature in maps made at the VLA. The $20 \mathrm{~cm}$ profile is seen to peak later than the higher frequencies, and shows less structure. The $20 \mathrm{~cm}$ radio burst (Figure 6(a)) shows a source predominantly immediately above the flare region on the disk, coincident with the $6 \mathrm{~cm}$ burst source (Figure 6(b), with the corresponding field of view indicated by a
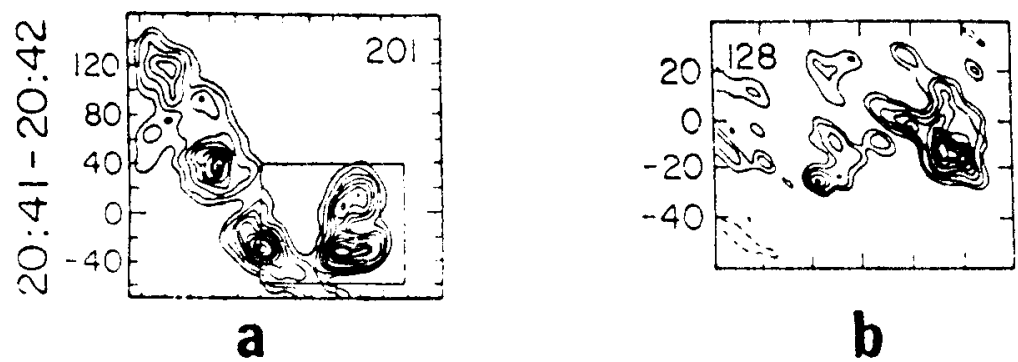

Fig. 6(a, b). The maps of the 20 and $6 \mathrm{~cm}$ burst sources, respectively, at one time $(20: 41-20: 42)$. Note that the $20 \mathrm{~cm}$ structure varied considerably during the burst. The box in (a) indicates the field of view of the $6 \mathrm{~cm}$ map in (b).

box on Figure 6(a)), together with an apparently much higher and weaker source well above the limb. The complex time profile of the flare is due to the appearance of multiple sources at both 6 and $20 \mathrm{~cm}$, which are apparent in Figures 6(a) and 6(b). In general, it is difficult to discuss the energetics of this event due to the complexity of the spatial structure. It is interpreted as the interaction of multiple loops in the active region, including some very high loops responsible for the $20 \mathrm{~cm}$ emission seen above the limb. Both $6 \mathrm{~cm}$ and $20 \mathrm{~cm}$ brightness temperatures exceed $10^{8} \mathrm{~K}$, and non-thermal emission is implied at both wavelengths.

Melozzi, Kundu, and Shevgaonkar (1985) also observed several bursts at both 6 and $20 \mathrm{~cm}$. They occurred in three groups spread over an hour of observations. The three bursts showed a range of behaviours, although coming from the same active region: all three were seen at $6 \mathrm{~cm}$, but one was completely absent at $20 \mathrm{~cm}$, one was a factor of 4 lower in flux at $20 \mathrm{~cm}$ compared to $6 \mathrm{~cm}$, and the third was stronger at $20 \mathrm{~cm}$ than at $6 \mathrm{~cm}$. In addition, the latter burst showed more fine time structure at $20 \mathrm{~cm}$ than at $6 \mathrm{~cm}$. In some of the bursts the $20 \mathrm{~cm}$ sources were larger and simpler in appearance than the $6 \mathrm{~cm}$ sources, but generally coincident, so that the $20 \mathrm{~cm}$ sources could have been outlining the tops of the loops with the $6 \mathrm{~cm}$ sources coming from lower down. The third burst referred to above seemed to be a double source at both wavelengths, but with the two wavelengths not coincident as if the $6 \mathrm{~cm}$ outlined one loop and the $20 \mathrm{~cm}$ another. The polarization structure of the maps was in agreement with this idea. We note that 'spike' bursts seem to occur more commonly at $20 \mathrm{~cm}$ than at $6 \mathrm{~cm}$ during the rise phase of flares but cannot be recognized without high-time-resolution equipment (e.g., see the review by Benz, 1986). Such spikes could well explain fine structure seen at $20 \mathrm{~cm}$ but not at $6 \mathrm{~cm}$. 


\section{Discussion}

For the average microwave burst the peak wavelength is around $4 \mathrm{~cm}$. For such a burst one does not expect to see the same structures at 6 and $2 \mathrm{~cm}$, since the shorter wavelength is optically thin and the longer wavelength is optically thick. Only when two wavelengths are both optically thin would one expect to see the same source structure, as indeed was the case in the 1980 October 1 event, since one is seeing the same electrons at both wavelengths. When both wavelengths are optically thick, the optically thick layer will be higher up for the longer wavelength. Since one is looking at two different layers, again one does not expect that the observed structure will be the same. The observations confirm this: usually the $20 \mathrm{~cm}$ burst sources are much larger than the $6 \mathrm{~cm}$ sources, and show different structures.

Thus, on the basis of the small number of examples available, we conclude that the assumption of a simple source structure usually used in the analysis of nonimaging radio data is occasionally adequate, in the sense that essentially the same population of electrons is producing the burst at the two wavelengths, if one is looking at two wavelengths close to the burst peak (in the solar case, 2 and $6 \mathrm{~cm}$ ). However, if one is looking at a wavelength far from the burst spectral peak, such as $20 \mathrm{~cm}$ in most solar bursts, then often other sources are present which may be unrelated to those at higher wavelengths. Conclusions made by comparing data at the two wavelengths in this case are clearly misleading.

For stellar observations of $\mathrm{M}$ dwarfs, these conclusions are not particularly relevant since it is well known that radio flares on these stars are due to an as yet unidentified coherent mechanism (e.g., Dulk, 1985). However, radio flares on RS CVn stars are probably due to non-thermal gyrosynchrotron emission, and thus are more similar to solar flares. If the frequency spectra of these flares are particularly simple, then the usual analysis is probably valid. That is, a single population of electrons is probably responsible for the emission at all wavelengths, and one can sensibly use information gained at different wavelengths to deduce information about that electron population. VLBI observations (Mutel et al., 1985) show that the size of the RS CVn radio sources is comparable to the size of the star, at least, and this implies that large loops trap the electrons. We have no information on the structure of these loops, and thus cannot say whether they are truly simple. That this is not the case for solar radio bursts needs to be borne in mind when interpreting stellar observations.

The VLA can now observe at $3.7 \mathrm{~cm}(8.4 \mathrm{GHz})$. What would we expect to see in solar observations at this wavelength based on this survey of previous observations? Since $8.4 \mathrm{GHz}$ is close to the typical peak of a non-thermal burst, it will most likely resemble the $6 \mathrm{~cm}$ optically thick source structure rather than the $2 \mathrm{~cm}$ optically thin source. However, in principle it should be smaller than a $6 \mathrm{~cm}$ source, since the latter is at a greater height, and one might hope to see a transition from optically thin to optically thick during the rise phase of a burst. 


\section{Acknowledgements}

This research was supported by NSF grant ATM-87-17157, NASA grant NAGW1541, and NASA contract NAG 5-969. The computions were done at the University of Maryland's Computer Science Center.

\section{References}

Batchelor, D. A., Crannell, C. J., Wiehl, H. J., and Magun, A.: 1985, Astrophys. J. 295, 258. Benz, A. O.: Solar Phys. 104, 99.

Dulk, G. A.: 1985, Ann. Rev. Astron. Astrophys. 23, 169.

Dulk, G. A., Bastian, T. S., and Kane, S. R.: 1986, Astrophys. J. 300, 438.

Kundu, M. R., Velusamy, T., and White, S. M.: 1987, Astrophys. J. 321, 593.

Melozzi, M., Kundu, M. R., and Shevgaonkar, R. K.: 1985, Solar Phys. 97, 345.

Mutel, R. L., Lestrade, J.-F., Preston, R. A., and Phillips, R. B.: 1985, Astrophys. J. 289, 262.

Shevgaonkar, R. K. and Kundu, M. R.: 1985, Astrophys. J. 292, 733.

Velusamy, T., Kundu, M. R., Schmahl, E. J., and McCabe, M.: 1987, Astrophys. J. 319, 984. 